\section{Xylazine Detection and Involvement in Drug Overdose Deaths — United States, 2019}

Mbabazi Kariisa, $\mathrm{PhD}^{1}$; Priyam Patel, $\mathrm{MSPH}^{1,2}$;

Herschel Smith, MPH ${ }^{1,2}$; Jessica Bitting, MS 1,3

Xylazine is a drug used in veterinary medicine as an animal sedative with muscle relaxant and analgesic properties (1). It is not approved by the Food and Drug Administration for use in humans, in whom it acts as a central nervous system depressant and can cause respiratory depression, slowed heart rate, and hypotension (2). When used as a toxic adulterant in illicitly produced opioids such as fentanyl or heroin (3), xylazine might potentiate sedation and respiratory depression, increasing the risk for fatal overdose. In addition, because xylazine is not an opioid, it does not respond to opioid reversal agents such as naloxone; therefore, if illicit opioid products containing xylazine are used, naloxone might be less effective in fully reversing an overdose. Several states have reported increases in xylazine-involved overdose deaths; however, the prevalence of xylazine involvement in drug overdose deaths (overdose deaths) has not been extensively studied, particularly in the United States (4). To better understand the impact of xylazine adulteration on the evolving drug overdose epidemic in the United States, CDC analyzed unintentional and undetermined intent overdose death data from the State Unintentional Drug Overdose Reporting System (SUDORS) in 38 states and the District of Columbia (DC).*, $\dagger$

A SUDORS case was defined as xylazine-positive if xylazine was detected on postmortem toxicology or if xylazine was listed on the death certificate as a contributing cause of death

\footnotetext{
*SUDORS captures data on fatal unintentional and undetermined intent overdoses. For all captured overdose deaths, SUDORS records all drugs detected by postmortem toxicology, even those not ruled by a medical examiner or coroner to have contributed to the death. A drug was recorded as contributing to death when the death certificate or medical examiner or coroner report listed the drug as a contributing factor.

$\dagger$ Thirty-eight states and DC reported data during January 2019-December 2019. Twenty-nine jurisdictions reported deaths that occurred during the entire period: Alaska, California, Connecticut, District of Columbia, Delaware, Georgia, Illinois, Indiana, Kentucky, Maine, Massachusetts, Minnesota, Missouri, Nevada, New Hampshire, New Jersey, New Mexico, North Carolina, Ohio, Oklahoma, Pennsylvania, Rhode Island, Tennessee, Utah, Vermont, Virginia, Washington, West Virginia, and Wisconsin. Four additional states only reported deaths that occurred during January-June 2019: Florida, Louisiana, Maryland, and Michigan. Six states only reported deaths that occurred during July-December 2019: Arizona, Colorado, Kansas, Montana, Oregon, and South Dakota. Thirty-one jurisdictions abstracted data on all drug overdose deaths within the jurisdiction and seven states (California, Florida, Illinois, Indiana, Louisiana, Missouri, and Washington) abstracted data on deaths within a subset of counties accounting for at least $75 \%$ of that state's overdose deaths in 2017, or at least 1,500 overdose deaths. Data were current as of December 10, 2020.
}

by the medical examiner or coroner based on postmortem toxicology detection, evidence of drug use at the scene, or witness reports of drug use. SUDORS cases in which xylazine is listed on the death certificate as a contributing cause of death by the medical examiner or coroner were defined as xylazine-involved. Thus, a xylazine-involved case would also be considered to be xylazine-positive by definition; however, a xylazine-positive case would not always mean that xylazine contributed to the death (i.e., xylazine-involved). Using data from 38 states and DC, CDC examined xylazine-positive and xylazine-involved overdose deaths that occurred during 2019. In addition, detailed narrative text for each case was reviewed for information about xylazine use or presence among drug products or paraphernalia found at the scene.

Among 45,676 overdose deaths reported to SUDORS during January-December 2019, xylazine-positive (826; 1.8\%), and xylazine-involved $(531 ; 1.2 \%)$ deaths were identified in 25 and 23 states, respectively. Xylazine was listed as a cause of death in $64.3 \%$ of deaths in which it was detected. The majority of xylazine-involved deaths were among males (73.1\%), non-Hispanic White persons (75.4\%), and from states in the Northeast Census region $(67.0 \%) .{ }^{\S}$ Among all xylazine-involved deaths, one or more other drugs, particularly illicit drugs, were also listed as a cause of death, and $98.7 \%$ of xylazine-positive deaths and $99.1 \%$ of xylazine-involved deaths had fentanyl (including analogs) listed as a cause of death. Cocaine and heroin were listed as a cause of death in $32.1 \%$ and $26.0 \%$ of xylazine-positive deaths respectively and in $29.6 \%$ and $28.4 \%$ of xylazine-involved deaths respectively (Table).

The findings in this report are subject to at least one limitation. Estimates of xylazine detection in overdose deaths might be underestimated. Data reviews revealed instances where xylazine presence was noted at the scene of the overdose but not detected on postmortem toxicology. Routine postmortem toxicology panels might not have included tests for xylazine, and current testing protocols for xylazine are not standard, which could result in missed detection (5).

During 2019, fewer than $2 \%$ of SUDORS overdose deaths from 38 states and DC were xylazine-positive. Xylazine contributed to death in approximately one half of deaths in which it was detected and was primarily co-involved with fentanyl. The detection of xylazine and its involvement in overdose deaths in multiple jurisdictions is concerning and warrants continued surveillance to inform overdose response and

\footnotetext{
$\$$ Connecticut, Maine, Massachusetts, New Hampshire, New Jersey, New York, Pennsylvania, Rhode Island, and Vermont.
} 
TABLE. Characteristics of drug overdose decedents with xylazine detected on postmortem toxicology (xylazine-positive) or listed as a cause of death (xylazine-involved) - State Unintentional Drug Overdose Reporting System, 38 states and the District of Columbia,* 2019

\begin{tabular}{|c|c|c|}
\hline \multirow[b]{2}{*}{ Characteristic } & \multicolumn{2}{|c|}{ Classification of deaths, no. (\%) } \\
\hline & $\begin{array}{c}\text { Xylazine-positive }^{\dagger} \\
(n=826)\end{array}$ & $\begin{array}{c}\text { Xylazine-involved } \$ \\
\quad(n=531)\end{array}$ \\
\hline \multicolumn{3}{|l|}{ Sex } \\
\hline Male & 602 (72.9) & $388(73.1)$ \\
\hline Female & $224(27.1)$ & $143(26.9)$ \\
\hline \multicolumn{3}{|l|}{ Raceף } \\
\hline White, non-Hispanic & $604(74.8)$ & $396(75.4)$ \\
\hline Black, non-Hispanic & $106(13.1)$ & $68(13.0)$ \\
\hline Hispanic & $90(11.1)$ & -** \\
\hline Other & $11(1.4)$ & 一** \\
\hline \multicolumn{3}{|l|}{ Age group, yrs } \\
\hline $15-24$ & $60(7.3)$ & $41(7.7)$ \\
\hline $25-34$ & $265(32.1)$ & $181(34.1)$ \\
\hline $35-44$ & $227(27.5)$ & $138(26.1)$ \\
\hline $45-54$ & $147(17.8)$ & $91(17.1)$ \\
\hline $55-64$ & $109(13.2)$ & -** \\
\hline$\geq 65$ & $18(2.2)$ & - ${ }^{* *}$ \\
\hline \multicolumn{3}{|l|}{ U.S. Census region ${ }^{+\dagger}$} \\
\hline Northeast & $568(68.8)$ & $356(67.0)$ \\
\hline Midwest & $144(17.4)$ & $91(17.1)$ \\
\hline South & $104(12.6)$ & - ** \\
\hline West & $10(1.2)$ & —** \\
\hline \multicolumn{3}{|c|}{ 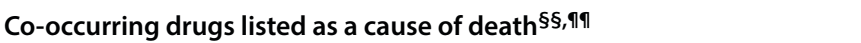 } \\
\hline $\begin{array}{l}\text { Any fentanyl } \\
\text { (including analogs) }\end{array}$ & $815(98.7)$ & $526(99.1)$ \\
\hline Heroin ${ }^{* * *}$ & $215(26.0)$ & $151(28.4)$ \\
\hline Benzodiazepines & $141(17.1)$ & 105 (19.8) \\
\hline Prescription opioids ${ }^{\dagger+\dagger}$ & $94(11.4)$ & $71(13.4)$ \\
\hline Cocaine & $265(32.1)$ & $157(29.6)$ \\
\hline Alcohol & $98(11.9)$ & $67(12.6)$ \\
\hline Methamphetamine & $102(12.4)$ & $62(11.7)$ \\
\hline
\end{tabular}

prevention efforts. Naloxone administration might not be as effective at fully reversing overdose-related signs and symptoms when xylazine and highly potent opioids such as fentanyl are present, although naloxone should always be administered. No pharmaceutical antidote is specific to xylazine, and immediate supportive care, especially respiratory and cardiovascular support, is critical in the event of an overdose when the presence of xylazine is suspected. Implementing routine standardized postmortem toxicology testing protocols for xylazine could help better elucidate the role of xylazine in drug overdose deaths.

\section{Acknowledgments}

Jurisdictions participating in CDC's Overdose Data to Action (OD2A) program and providing data to SUDORS, including state and jurisdictional health departments, vital registrar offices, and coroner and medical examiner offices; Christina A. Mikosz, Division of Overdose Prevention, National Center for Injury Prevention and Control, CDC; CDC OD2A team, Division of Overdose Prevention, National Center for Injury Prevention and Control, CDC.
TABLE. (Continued) Characteristics of drug overdose decedents with xylazine detected on postmortem toxicology (xylazine-positive) or listed as a cause of death (xylazine-involved) - State Unintentional Drug Overdose Reporting System, 38 states and the District of Columbia, 2019

Abbreviation: SUDORS = State Unintentional Drug Overdose Reporting System.

* Thirty-nine jurisdictions reported data during January-December 2019, including 29 that reported deaths that occurred during the entire period: Alaska, California, Connecticut, District of Columbia, Delaware, Georgia, Illinois, Indiana, Kentucky, Maine, Massachusetts, Minnesota, Missouri, Nevada, New Hampshire, New Jersey, New Mexico, North Carolina, Ohio, Oklahoma, Pennsylvania, Rhode Island, Tennessee, Utah, Vermont, Virginia, Washington, West Virginia, and Wisconsin. Four additional states only reported deaths that occurred during January-June 2019: Florida, Louisiana, Maryland, and Michigan. Six states only reported deaths that occurred during July-December 2019: Arizona, Colorado, Kansas, Montana, Oregon, and South Dakota. Thirty-one jurisdictions abstracted data on all drug overdose deaths within the jurisdiction and seven states (California, Florida, Illinois, Indiana, Louisiana, Missouri, and Washington) abstracted data on deaths within a subset of counties accounting for at least $75 \%$ of that state's overdose deaths in 2017, or at least 1,500 overdose deaths. Data were current as of December 10, 2020.

${ }^{\dagger}$ A SUDORS case was defined as xylazine-positive if xylazine was detected on postmortem toxicology or if xylazine was listed as a contributing cause of death by the medical examiner or coroner on the death certificate. The medical examiner or coroner determines if xylazine was involved or contributed to the death based on postmortem toxicology detection, evidence of drug use at the scene or witness reports of drug use.

$\S$ SUDORS cases that had xylazine listed as a contributing cause of death by the medical examiner or coroner were defined as xylazine-involved. Thus, a xylazine-involved case would also be considered to be xylazine-positive by definition; however, a xylazine-positive case would not always mean that xylazine contributed to the death (i.e., xylazine-involved).

" Race and ethnicity data were missing for 15 xylazine-positive decedents. Race and ethnicity data were missing for six xylazine-involved decedents.

${ }^{* *}$ Cells with $\leq 9$ deaths are not reported. Some cells are not reported to prevent calculation of another suppressed cell.

${ }^{+\dagger}$ Northeast: Connecticut, Maine, Massachusetts, New Hampshire, New Jersey, New York, Pennsylvania, Rhode Island, and Vermont; Midwest: Illinois, Indiana, lowa, Kansas, Michigan, Minnesota, Missouri, Nebraska, North Dakota, Ohio, South Dakota, and Wisconsin; South: Alabama, Arkansas, Delaware, District of Columbia, Florida, Georgia, Kentucky, Louisiana, Maryland, Mississippi, North Carolina, Oklahoma, South Carolina, Tennessee, Texas, Virginia, and West Virginia; West: Alaska, Arizona, California, Colorado, Hawaii, Idaho, Montana, Nevada, New Mexico, Oregon, Utah, Washington, and Wyoming.

$\S \S$ Identified as a cause of death by a medical examiner or coroner.

"१ Multiple drugs could be listed as a cause of death; therefore, drugs are not mutually exclusive.

*** Drugs coded as heroin were heroin and 6-monoacetylmorphine. In addition, morphine was coded as heroin if detected along with 6-acetylmorphine or if scene, toxicology, or witness evidence indicated presence of heroin impurities or other illicit drugs, injection, illicit drug use, or a history of heroin use.

${ }^{t+\dagger}$ Drugs coded as prescription opioids were alfentanil, buprenorphine, codeine, dextrorphan, hydrocodone, hydromorphone, levorphanol, loperamide, meperidine, methadone, morphine, noscapine, oxycodone, oxymorphone, pentazocine, prescription fentanyl, propoxyphene, remifentanil, sufentanil, tapentadol, and tramadol. Also included as prescription opioids were brand names (e.g., Opana) and metabolites (e.g., nortramadol) of these drugs and combinations of these drugs and nonopioids (e.g., acetaminophen-oxycodone). Morphine was included as prescription only if scene or witness evidence did not indicate likely heroin use and if 6-acetylmorphine was not also detected. Fentanyl was coded as a prescription opioid based on scene, toxicology, or witness evidence. 
Corresponding author: Mbabazi Kariisa, mkariisa@cdc.gov.

${ }^{1}$ Division of Overdose Prevention, National Center for Injury Prevention and Control, CDC; ${ }^{2}$ Oak Ridge Institute for Science and Education, Oak Ridge, Tennessee; ${ }^{3}$ National Network of Public Health Institutes, New Orleans, Louisiana.

All authors have completed and submitted the International Committee of Medical Journal Editors form for disclosure of potential conflicts of interest. No potential conflicts of interest were disclosed.

\section{References}

1. Reyes JC, Negrón JL, Colón HM, et al. The emerging of xylazine as a new drug of abuse and its health consequences among drug users in Puerto Rico. J Urban Health 2012;89:519-26. PMID:22391983 https://doi. org/10.1007/s11524-011-9662-6
2. Ruiz-Colón K, Chavez-Arias C, Díaz-Alcalá JE, Martínez MA. Xylazine intoxication in humans and its importance as an emerging adulterant in abused drugs: a comprehensive review of the literature. Forensic Sci Int 2014;240:1-8. PMID:24769343 https://doi.org/10.1016/j. forsciint.2014.03.015

3. Wong SC, Curtis JA, Wingert WE. Concurrent detection of heroin, fentanyl, and xylazine in seven drug-related deaths reported from the Philadelphia Medical Examiner's Office. J Forensic Sci 2008;53:495-8. PMID:18284526 https://doi.org/10.1111/j.1556-4029.2007.00648.x

4. Johnson J, Pizzicato L, Johnson C, Viner K. Increasing presence of xylazine in heroin and/or fentanyl deaths, Philadelphia, Pennsylvania, 2010-2019. Inj Prev 2021;27:395-8. PMID:33536231 https://doi.org/10.1136/ injuryprev-2020-043968

5. Ruiz-Colón K, Martínez MA, Silva-Torres LA, et al. Simultaneous determination of xylazine, free morphine, codeine, 6-acetylmorphine, cocaine and benzoylecgonine in postmortem blood by UPLC-MS-MS. J Anal Toxicol 2012;36:319-26. PMID:22582266 https://doi. org/10.1093/jat/bks024 